\title{
EVOLUTION OF THE PROGENITOR OF SN 1987A IN THE HR DIAGRAM
}

\author{
H. YAMAOKA ${ }^{1}, \mathrm{H} . \mathrm{SAIO}^{1}, \mathrm{~K} . \mathrm{NOMOTO}^{1}$, and M. KATO ${ }^{2}$ \\ ${ }^{1}$ Department of Astronomy, University of Tokyo \\ ${ }^{2}$ Department of Astronomy, Keio University
}

We calculate the blue-red-blue evolution of the progenitor of SN 1987A in the HR diagram by adopting the Schwarzschild criterion for convection and by choosing appropriate parameters for mass loss and mixing [Fig. 1 (left) where metallicity is $Z=0.005]$. During helium burning, the star moves from the blue to the red due to mass loss from $23 M_{\odot}$ to $16 M_{\odot}$. Figure 2 (right) shows the lifetime in effective temperature bins normalized to unity (solid) compared with the number of supergiants with $-8<M_{\mathrm{bol}}<.-9$ in the LMC, which is also normalized to unity (dashed). The model is qualitatively consistent with the observed histogram.

If the Ledoux criterion is adopted, the star ignites helium in the red supergiant stage, making a loop, and eventually returns to red. The timescale for the star to move from the blue to the red seems to be too fast to reproduce the observed histogram.

The evolution from the red to the blue in Fig. 1 takes place when the surface helium mass fraction is enhanced to $Y=0.43$. Because of decreased opacity due to low $Z$ and high $Y$, the surface luminosity exceeds the core luminosity. To compensate this luminosity imbalance, the envelope contracts back to the blue. The deep mixing model can naturally explain the large $Y$ and N/C and N/O ratios observed in the circumstellar materials.
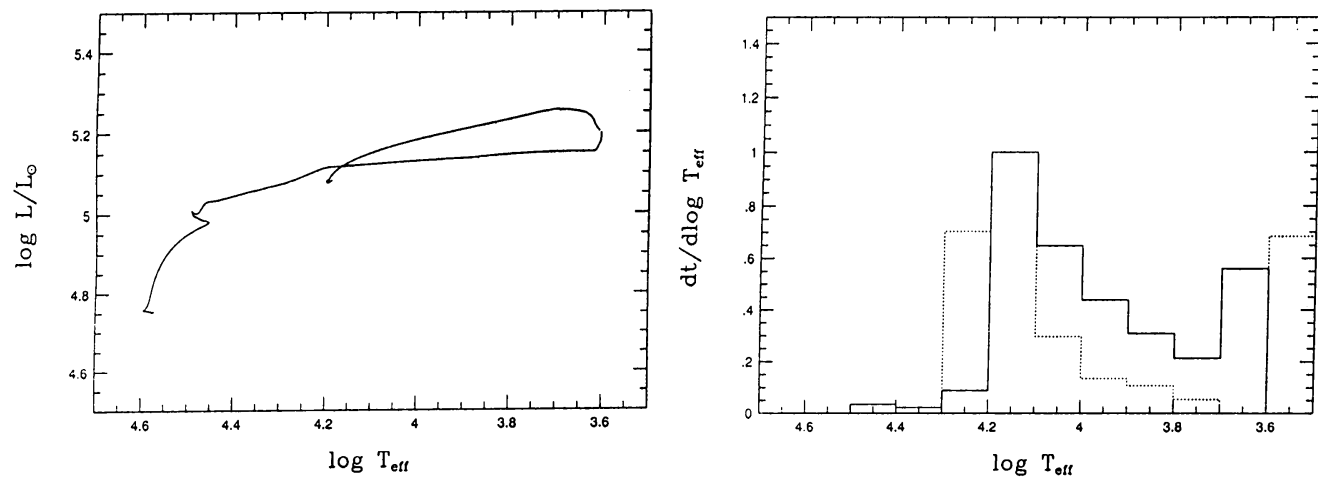

571

K. A. van der Hucht and B. Hidayat (eds.),

Wolf-Rayet Stars and Interrelations with Other Massive Stars in Galaxies, 571.

(C) 1991 IAU. Printed in the Netherlands. 


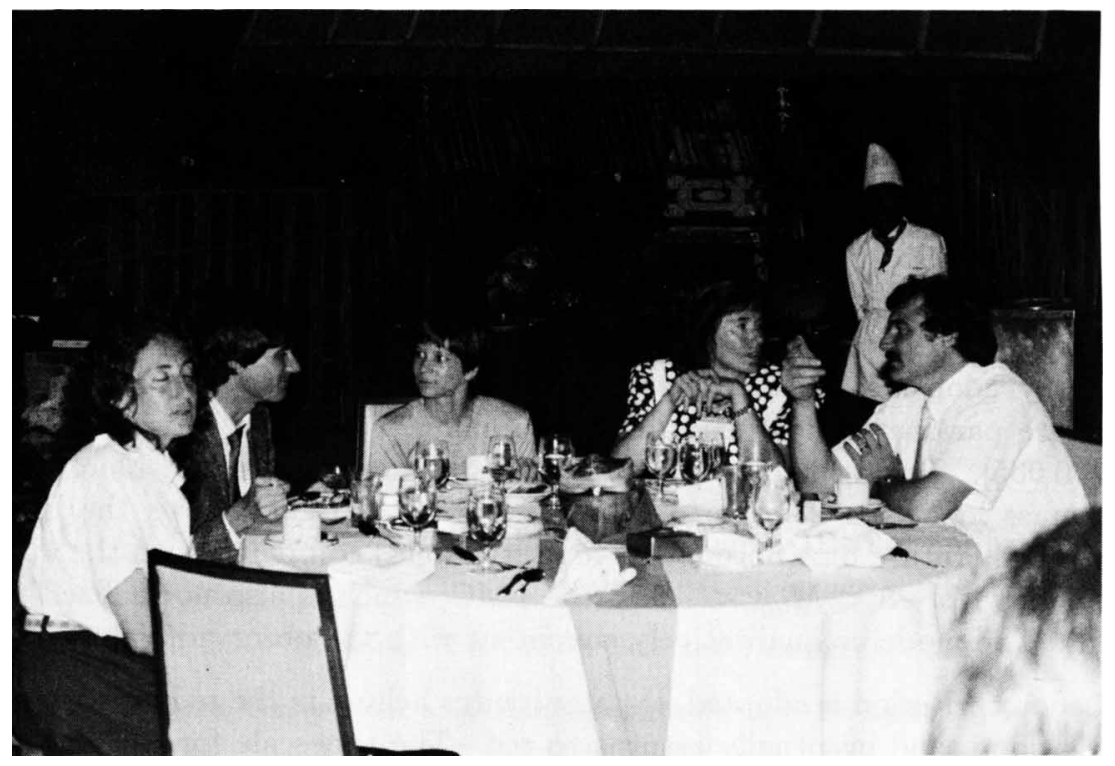

Koenigsberger, Filippenko, Taylor, Schulte-Ladbeck, Hillier 